\title{
Nos bactéries \\ commensales
}

\section{Un coup de pouce pour les thérapies antitumorales}

> Deux travaux dont les résultats ont été publiés récemment dans la revue Science montrent que le microbiote joue un rôle clé dans la réponse à différentes thérapies antitumorales. IIs mettent ainsi en garde contre l'administration non raisonnée d'antibiotiques à des patients recevant des traitements anticancéreux. <

Le tube digestif des mammifères contient environ 100 billions $\left(10^{14}\right)$ de bactéries commensales bénéfiques - plus de dix bactéries pour chaque cellule de notre organisme! Cette flore intestinale joue un rôle vital dans le bon fonctionnement du système immunitaire, qui a « appris » a coexister de façon pacifique avec les populations bactériennes com-

$(\rightarrow)$ Voir la Brève de N. Korneychuk, $m / s n^{\circ} 5$, mai 2013, page 479 mensales [1] $(\rightarrow)$.

Des données récentes montrent que le microbiote aide à contrôler l'inflammation au niveau local. Pas très surprenant donc que des déséquilibres de la flore intestinale (ou dysbiose) puissent favoriser un état inflammatoire chronique, qui contribue à l'apparition de cancers colorectaux [2-4]. De façon un peu moins attendue, le microbiote peut aussi influencer des réponses immunes en dehors du tractus digestif. Ainsi, des souris traitées par des antibiotiques (et dont la flore intestinale est donc fortement réduite) sont peu protégées lors d'infections virales pulmonaires en raison d'un défaut de production d'interférons de type I et d'une diminution de l'activation des cellules natural killer (NK) [5] et des macrophages [6] $(\rightarrow)$.

$(\rightarrow)$ Voir la Brève de N. Korneychuk, $\mathrm{m} / \mathrm{s} \mathrm{n}^{\circ}$ 5, mai 2013, page 479

Le microbiote peut-il alors moduler d'autres réactions inflammatoires et immunes «à distance », par exemple celles qui accompagnent des tumeurs hors du système digestif ? C'est la question qu'a explorée une équipe américaine [7] et une équipe française [8], et leurs résultats ont été publiés récemment dans Science. Nous les détaillons ci-dessous.

Cet article fait partie du numéro thématique publié par médecine/sciences en avril 2014 et intitulé « Microenvironnement tumoral ».

\section{Adelaida Sarukhan}

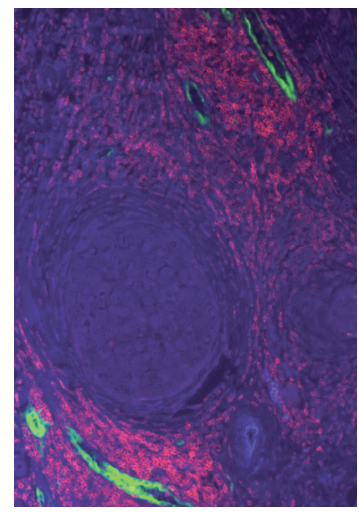

CRI Inserm,

C. Enric Gimenez 17-19, 08034 Barcelona, Espagne. adelaida.sarukhancasamitjana@inserm.fr

\section{La flore intestinale module}

le microenvironnement des tumeurs

Les cellules immunes présentes dans les tumeurs induisent une inflammation chronique de faible intensité qui favorise la prolifération des cellules tumorales et la suppression des réponses immunes $[9,10](\rightarrow)$.

Ce microenvironnement suppresseur de la tumeur peut être altéré par certaines thérapies anticancéreuses, favorisant l'induction de réponses antitumorales efficaces [11-13] $(\rightarrow)$.

$(\rightarrow)$ Voir les Synthèses de W.H. Fridman et C. Sautès-Fridman, et de M. Bruchard et F. Ghiringhelli, pages 359 et 429 de ce numéro

$(\rightarrow)$ Voir la Synthèse de J. Galon et al., page 439 de ce numéro

Pour déterminer si la flore intestinale joue un rôle dans ce processus, l'équipe de G. Trinchieri et R. Goldszmid a administré par voie orale à des souris une combinaison d'antibiotiques afin d'éliminer la flore intestinale; différents types de cellules tumorales murines ont ensuite été greffés aux souris par voie sous-cutanée [7]. Dans les tumeurs des souris traitées par antibiotiques, l'expression de gènes intervenant dans l'inflammation, la phagocytose et la présentation antigénique était diminuée, alors que l'expression de gènes liés au métabolisme, à la régénération tissulaire et au cancer était augmentée. Les auteurs ont ensuite analysé l'effet des antibiotiques sur la réponse à deux types de thérapies antitumorales (Figure 1). La première est une immunothérapie basée sur l'activation (par des oligodéoxynucléotides $\mathrm{CpG}$ ) du récepteur TLR9 (toll like receptor) présent sur les cellules immunes innées (dont les cellules myéloïdes), et le blocage (par un anticorps) du récepteur de I'IL (interleukine)-10, une cytokine immunosuppressive. Cette immunothérapie induit une nécrose de la tumeur via la production de TNF $\alpha$ (tumor necrosis factor $\alpha$ ) par les cellules myéloïdes intratumorales, 


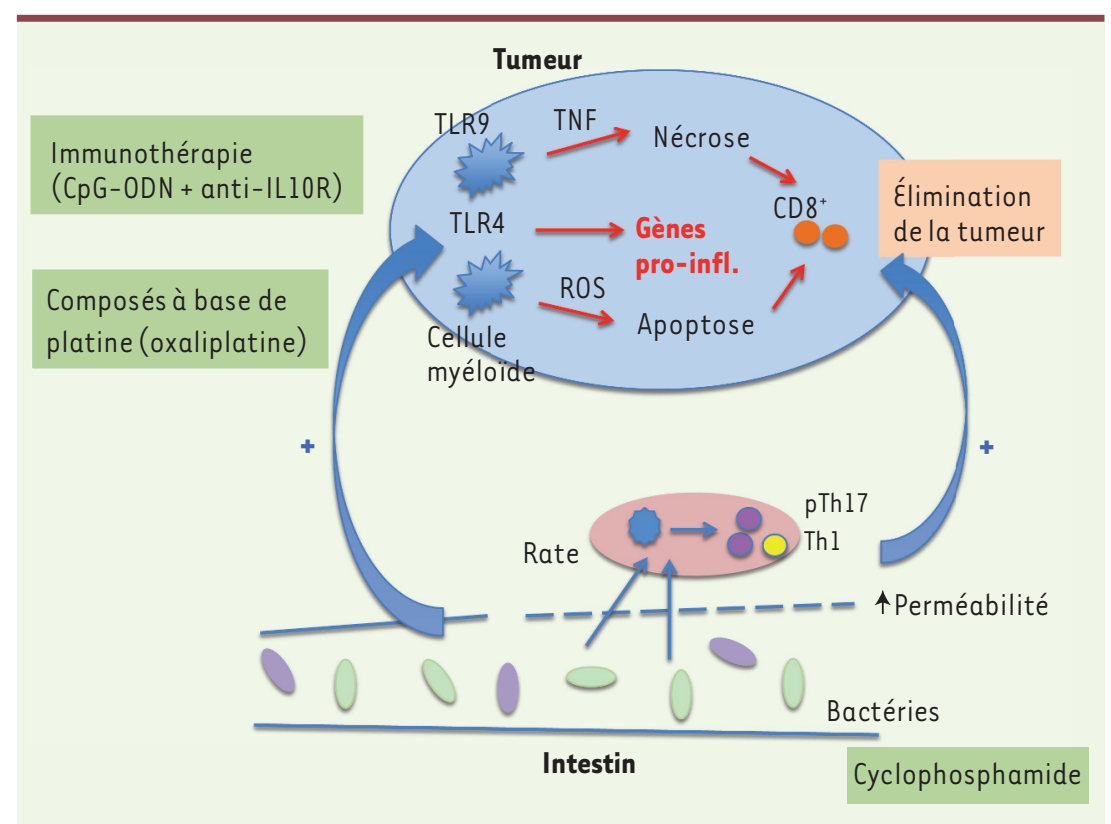

Figure 1. L'efficacité des thérapies antitumorales est influencée par l'action des bactéries commensales. Des produits dérivés de bactéries commensales à Gram positif et à Gram négatif activent des cellules myéloödes dans les tumeurs, augmentant ainsi leur production de TNF lors d'une immunothérapie, ou celle des ROS lors d'une chimiothérapie à base d'oxilaplatine. II en résulte une surexpression de gènes proinflammatoires (pro-infl.) et la génération et/ ou l'activation de cellules T CD $8^{+}$cytotoxiques. Le cyclophosphamide induit une augmentation de la perméabilité intestinale et la translocation de bactéries à Gram positif vers la rate et les ganglions mésentériques, où elles favorisent la génération de cellules Thl et pTh17 antitumorales. $C p G-O D N$ : oligodéoxynucléotides synthétiques (ODN) contenant des motifs CPG ; ILIOR: anticorps anti-récepteur de I'IL-10;

TLR9 : Toll like receptor 9, il reconnaît des séquences CpG non méthylées de l'ADN; TLR4: Toll like receptor 4, il reconnaît le lipopolysaccharide (LPS) des bactéries à Gram négatif ; TNF : facteur de nécrose tumorale; ROS : dérivés réactifs de l'oxygène.

suivie par une réponse T CD8 cytotoxique qui élimine la tumeur [14]. La deuxième est une chimiothérapie à base d'oxaliplatine, couramment utilisée chez l'homme et dont l'effet ne dépend pas directement d'une réaction inflammatoire (avec activation de cellules immunes innées), mais de l'apoptose des cellules tumorales et de l'induction d'une immunité T CD8 cytotoxique [15].

Les deux types de thérapies s'avéraient moins efficaces chez les souris sous antibiotiques, comme en témoignaient une progression de la taille des tumeurs et une mortalité 3 à 4 fois plus élevée que chez les souris qui n'avaient pas reçu d'antibiotiques. De plus, l'expression de gènes codant pour des protéines anti-inflammatoires observée dans les tumeurs des souris contrôles (soumises aux mêmes thérapies anticancéreuses mais sans antibiotiques), était fortement diminuée chez les souris traitées par des antibiotiques ou sans flore intestinale (axéniques).

Les auteurs ont ensuite déterminé que la production de TNF $\alpha$ par les cellules myéloïdes intratumorales en réponse à l'immunothérapie nécessitait une stimulation des TLR4 [1] par des produits de la flore intestinale (tels que le LPS, lipopolysaccharide, produit de la paroi bactérienne). Ces derniers sont très diminués chez les souris sous antibiotiques, et, en conséquence, l'activation de TLR4 et les niveaux de TNF $\alpha$ dans les tumeurs également. De fait, les auteurs ont corrélé la production de TNF $\alpha$ dans la tumeur à la composition du microbiote et à la présence dans l'intestin de certaines espèces de bactéries à Gram négatif (Alistipes) mais aussi à Gram positif (Ruminococcus).

À la différence de l'immunothérapie, la réponse à l'oxaliplatine ne dépend pas du TNF, mais de la production de dérivés réactifs de l'oxygène (ROS) par les cellules myéloïdes dans la tumeur. L'activation de ces cellules est nécessaire (de fait, la réponse à l'oxaliplatine est caractérisée par une surexpression de gènes liés à l'activation, la fonction et la différenciation de monocytes), mais elle est indé- pendante des TLR4. Les antibiotiques agissant dès les premiers jours, les auteurs suggèrent que les bactéries commensales augmentent, en quelque sorte, la toxicité des molécules de platine, indépendamment de la réponse immune induite par la mort cellulaire, qui, elle, survient plus tardivement.

\section{La chimiothérapie favorise la translocation de certaines bactéries commensales}

Les mécanismes par lesquels les bactéries commensales peuvent, depuis l'intestin, avoir un effet sur le microenvironnement de tumeurs qui sont loin du tube digestif, restent encore à définir. Néanmoins, l'étude publiée dans le même numéro de Science par l'équipe de Laurence Zitvogel contribue à identifier le mécanisme par lequel les bactéries de l'intestin peuvent augmenter les réponses antitumorales lors d'une chimiothérapie.

Dans cette étude, les auteurs ont exploré l'effet du cyclophosphamide (CTX), une drogue anticancéreuse utilisée couramment, sur le microbiote et les réponses antitumorales. Ils avaient en effet remarqué que les patients recevant du CTX présentaient des troubles digestifs. Cette même équipe a précédemment montré que le CTX induit une mort cellulaire immunogénique, et stimule les lymphocytes Thl et Thl7 cells qui contrôlent la croissance tumorale ([16], voir aussi $[9,11]$ ). L'exploration du lien entre microbiote et CTX chez la souris montre que le CTX modifie la structure des villosités intestinales et de l'épithélium intestinal et augmente la perméabilité de la 
barrière épithéliale intestinale, provoquant la translocation de certaines bactéries commensales vers les ganglions mésentériques et la rate. La majorité des espèces cultivées à partir de ces organes lymphoïdes étaient Gram positives (avec une prédominance de Lactobacillus johnsonii et Enterococcus hirae). Ce passage bactérien était suivi de changements importants dans la composition de la flore intestinale, également observés chez les patients traités par CTX. Cette dysbiose s'accompagnait d'une conversion, dans la rate, de lymphocytes T CD4 en cellules Thl, cellules productrices d'IL17 (Th17) et cellules pathogéniques Th17 (pTh17), caractérisées par la coexpression d'IFN $\gamma$ (interféron) et du facteur de transcription Tbet. Cette augmentation dépend des bactéries commensales, car elle n'était pas observée chez des souris axéniques ou traitées avec une combinaison d'antibiotiques ou avec de la vancomycine, qui tue des bactéries à Gram positif. De plus, la production d'IL17 dans la rate était positivement corrélée à la présence de Lactobacillus dans l'intestin, et était détectée chez des souris naïves (non manipulées) recevant par gavage une solution de $L$. johnsonii et $\varepsilon$. hirae. Sur la base de ces résultats, les auteurs proposent que la translocation de certaines souches de bactéries commensales Gram positives dans les organes lymphoïdes est nécessaire et suffisante pour y induire la conversion de cellules T CD4 ${ }^{+}$ naïves en cellules pTh17 et Thl à forte activité antitumorale. Comme on pouvait s'y attendre, l'administration d'antibiotiques à des souris porteuses de différents types de tumeurs a fortement diminué leur réponse au CTX : cela se traduisait par un accroissement de la taille des tumeurs et une chute du nombre de cellules pTh17 et THl par rapport aux souris contrôles (n'ayant pas reçu d'antibiotiques). De façon importante, l'effet du CTX était restauré par l'injection de cellules pTh17, ce qui démontre le lien de cause à effet entre l'effet de la CTX sur la perméabilité intestinale et donc les mouvements du microbiote, la génération de cellules pTh17, et la réponse antitumorale.

\section{Conclusion}

Il est encore trop tôt pour exploiter ces observations et manipuler le microbiote humain afin d'améliorer les traitements anticancéreux. D'autres études sont nécessaires pour mieux comprendre les mécanismes par lesquels le microbiote régule les réponses inflammatoires (en particulier les cellules myéloïdes) dans et en dehors de l'intestin. Néanmoins, dans l'ensemble, ces résultats illustrent l'interaction complexe entre flore intestinale, inflammation, cancer et réponse thérapeutique. Ils prônent une utilisation rationnelle d'antibiotiques chez les patients cancéreux et soulignent l'importance de prendre en compte le microbiote comme un autre paramètre prédictif de la réponse aux thérapies antitumorales. $\diamond$

\section{SUMMARY}

\section{The intestinal microbiota: a boost for antitumor treatments}

Two new studies in mice, published in Science, show that the commensal bacteria populations in the gut play a key role in boosting responses to different antitumor regimens. These results argue for a rational use of antibiotics when managing infections in patients undergoing cancer therapies. $\diamond$
LIENS D’INTÉRÊT

L'auteur déclare n'avoir aucun lien d'intérêt concernant les données publiées dans cet article.

\section{RÉFÉRENCES}

1. Hooper LV, Macpherson AJ. Immune adaptations that maintain homeostasis with the intestinal microbiota. Nat Rev Immunol $2010 ; 10$ : 159-69.

2. Grivennikov SI, Wang K, Mucida D, et al. Adenoma-linked barrier defects and microbial products drive IL-23/IL-17-mediated tumour growth. Nature $2012 ; 491: 254-8$.

3. Wu S, Rhee KJ, Albesiano $\varepsilon$, et al. A human colonic commensal promotes colon tumorigenesis via activation of T helper type $17 \mathrm{~T}$ cell responses. Nat Med 2009 ; $15: 1016-22$.

4. Jobin C. Microbiome : un nouveau facteur de risque de cancer colorectal ? Med Sci (Paris) $2013 ; 29: 582-585$

5. Ganal SC, Sanos SL, Kallfass C, et al. Priming of natural killer cells by nonmucosal mononuclear phagocytes requires instructive signals from commensal microbiota. Immunity 2012 ; 37 : 171-86.

6. Abt MC, Osborne LC, Monticelli LA, et al. Commensal bacteria calibrate the activation threshold of innate antiviral immunity. Immunity $2012 ; 37$ : 158-70.

7. lida N, Dzutsev A, Stewart CA, et al. Commensal bacteria control cancer response to therapy by modulating the tumor microenvironment. Science $2013 ; 342: 967-70$.

8. Viaud S, Saccheri F, Mignot G, et al. The intestinal microbiota modulates the anticancer immune effects of cyclophosphamide. Science $2013 ; 342$ : 971-6.

9. Fridman WH, Sautès-Fridman C. Le microenvironnement tumoral : matrice nourricière, champ de bataille et cible thérapeutique des cancers. Med Sci (Paris) $2014 ; 30: 359-65$.

10. Bruchard M, Ghiringhelli F. Microenvironnement tumoral : cellules régulatrices et cytokines immunosuppressives. Med Sci (Paris) $2014 ; 30: 429-35$.

11. Galon J, Bindea G, Mlecnik B, et al. Microenvironnement immunitaire et cancer : intérêt de l'Immunoscore pour prédire l'évolution clinique. Med Sci (Paris) $2014 ; 30: 439-44$.

12. Apetoh L, Ghiringhelli F, Tesniere A, et al. Toll-like receptor 4-dependent contribution of the immune system to anticancer chemotherapy and radiotherapy. Nat Med $2007 ; 13: 1050-9$.

13. Shirota Y, Shirota H, Klinman DM. Intratumoral injection of CpG oligonucleotides induces the differentiation and reduces the immunosuppressive activity of myeloid-derived suppressor cells. J Immunol $2012 ; 188: 1592-9$.

14. Guiducci C, Vicari AP, Sangaletti S, Trinchieri G, Colombo MP. Redirecting in vivo elicited tumor infiltrating macrophages and dendritic cells towards tumor rejection. Cancer Res $2005 ; 65: 3437-46$.

15. Ghiringhelli F, Apetoh L, Tesniere A, et al. Activation of the NLRP3 inflammasome in dendritic cells induces IL-lbeta-dependent adaptive immunity against tumors. Nat Med $2009 ; 15: 1170-8$.

16. Viaud S, Flament C, Zoubir M, et al. Cyclophosphamide induces differentiation of Th17 cells in cancer patients. Cancer Res 2011 ; 71, 661-5.

TIRÉS À PART

A. Sarukhan

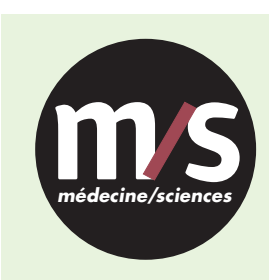

Abonnez-vous à médecine/sciences

Bulletin d'abonnement page 470 dans ce numéro de $\mathrm{m} / \mathrm{s}$ 